\title{
Superstring perturbation theory
}

\author{
Ido Adam
}

Received: 26 November 2008 / Accepted: 17 December 2008 / Published online: 11 February 2009

(C) The Author(s) 2009. This article is published with open access at Springerlink.com

\begin{abstract}
The state of superstring perturbation theory is reviewed with an emphasis on the state of the pure spinor superstring perturbation theory. We begin with a brief summary of the state of perturbation theory in the Ramond-Neveu-Schwarz and in the Green-Schwarz formulations of the superstring. Then we proceed to a quick review of the minimal and non-minimal pure spinor formulations of the superstring and discuss the multi-loop amplitude prescriptions in each of them. We end with a summary and open questions.
\end{abstract}

Keywords Superstring perturbation theory - The pure spinor superstring

\section{Introduction}

Superstring theories are claimed to be candidates for the ultimate quantum theory unifying all the interactions including gravity. In order to serve such a role it must be shown that superstring amplitudes are not plagued by the infinities that make field theory unsuitable to serve in such a role. In order to justify such claims the perturbation theory of the superstring must be defined and its structure of possible infinities analyzed.

There are three formulations of superstring theory. The first one is the GreenSchwarz superstring, whose main feature is manifest space-time supersymmetry. The second is the Ramond-Neveu-Schwarz formalism, in which space-time

This is a contribution to the proceedings of the WE-Heraeus-seminar, Quantum gravity: challenges and perspectives, dedicated to the memory of John A. Wheeler.

I. Adam (ه)

Max Planck Institute for Gravitational Physics (Albert Einstein Institute), Potsdam, Germany

e-mail: Ido.Adam@aei.mpg.de 
supersymmetry is not manifest but it has a larger symmetry on its world-sheet and whose quantization is generally more tractable then the Green-Schwarz superstring. Finally, the pure spinor formulation features space-time supersymmetry and is more easily quantized as well.

Here we very briefly summarize the status of perturbation theory in the RamondNeveu-Schwarz and the Green-Schwarz superstring and then concentrate on the pure spinor formulation of the superstring (mainly due to the author's bias).

None of the results presented here are claimed to be original and as superstring perturbation theory has been the subject of research for quite a long time the list of references is far from being complete and exhausting.

\section{The Ramond-Neveu-Schwarz and the Green-Schwarz superstrings}

\subsection{The Ramond-Neveu-Schwarz superstring}

Unlike the bosonic string, for which the path integral measure is fully known from first principles and in principle can be used to compute amplitudes to any desired order, the situation with the Ramond-Neveu-Schwarz (RNS) formulation of the superstring is less clear.

On general grounds the measure for the RNS superstring should include path integration over the matter fields as well as the ghosts, an integration over the super-moduli space - a space parameterizing the set of inequivalent super-Riemann surfaces of genus $g$ and $N$ marked points (the positions of the vertex operators on the Riemann surface), which is what remains after integrating out the world-sheet metric and gravitino, and a sum over all the possible spin structures for world-sheet fermions on a Riemann surface of genus $g$ with $N$ marked points. Additionally, there should be $b$-ghost and $\beta$-ghost insertions for each of the Grassmann-even and Grassmann-odd moduli, which can be chosen to include the positions of the vertex operators. (See [1] for a short pedagogical overview).

The measure for loop amplitudes of Type II superstrings was obtained in [2] and so was the one-loop measure for the heterotic string [3]. Both obtained it by gauge fixing the non-dynamic world-sheet supergravity of the superstring and obtaining the measure on the residual finite-dimensional super-moduli space. The issue of the unitary of the resulting perturbation series was addressed in [4] by showing its equivalence with the series obtained by using the light-cone gauge, which is manifestly unitary.

Naively, the one-loop superstring amplitude obtained from the measures above diverges for real momenta. The appropriate analytic continuation required for obtaining a finite well-defined Type II superstrings one-loop amplitude was found in [5].

Difficulties and ambiguities were encountered in the extension of the measure to arbitrary loops because of the difficulty in implementing appropriately the gaugefixing of the world-sheet superconformal symmetry. Based on methods proposed for gauge-fixing in $[6,7]$ a prescription for the two-loop amplitude was derived for even spin structures of two-loop amplitudes which was independent of the choice of the gauge slice and that after summation over the spin structures did not involve total derivatives on the super-moduli space [8-13]. Using this the one-, two- and 
three-point amplitudes for the bosonic Neveu-Schwarz states were shown to vanish while results for the four-point amplitudes indicated no two-loop corrections to the $R^{4}$ terms in the Type II effective action and to $F^{2} F^{2}, F^{2} R^{2}$ and $R^{4}$ in the heterotic string effective action [13]. However, the construction was specific to the two-loop case and was not amenable to extension to higher loop amplitudes.

In an attempt to generalize the amplitude prescription to higher genera two alternative two-loop measures were suggested based on the requirement of it being a modular form [14]. These two ansatze were more amenable to extension to higher genera. Using the requirements of holomorphy, factorization and modular invariance a proposal was put forward for the way to find the measure of the three-loop amplitude [15] provided that a certain mathematical relation was satisfied. This program for three-loop amplitude derivation was further developed in [16], where by relaxing some of the requirements, it was managed to find a measure satisfying the relaxed requirements and verified that the cosmological constant vanishes using this prescription.

Various attempts to extend D'Hoker and Phong's program to higher genera have been made. Among them are the ones by $[17,18]$ which proposed a four-loop measure and the latter even suggested a candidate for higher genera and they were verified to meet the non-renormalization requirements for such measures on a hyperelliptic locus of the moduli space [19]. However, [20] showed that according to these proposals the three-point function fails to vanish as expected from non-renormalization theorems and suggested a possible remedy. Moreover, in [21] it was shown that the cosmological constant does not vanish at genus five when this ansatz is used-implying that this ansatz as it is fails to meet a key requirement from the amplitudes.

Hence, the form of the RNS superstring measures is still an open question and subject to ongoing research.

\subsection{The Green-Schwarz superstring}

The Green-Schwarz superstring is a manifestly space-time super-Poincaré invariant formulation of the superstring. However, due to the existence of mixed first- and second-class constraints, it has been quantized only in the light-cone gauge, which breaks the manifest Poincaré symmetry and hence makes the computation of amplitudes non-covariant even though the final results should be Lorentz invariant in ten dimensions.

The theory of interacting Green-Schwarz superstrings was formulated by constructing the superstring field vertices [22-24] for the Type I and Type II superstring theories. This was done in the light-cone gauge, which broke the $S O(1,9)$ Lorentz symmetry leaving just a $S O(8)$ subgroup manifest and this subgroup was further broken to simplify the treatment. Thus Lorentz invariance was not manifest.

Moreover, by requiring that the super-Poincaré algebra close and that the tree-level amplitudes be finite it was found that additional contact terms appearing as counterterms in the Green-Schwarz Hamiltonian must be put when superstring vertex operators coincide [25-27]. The finite supersymmetric results for tree-level amplitudes obtained before required an analytic continuation of the momenta to unphysical regions in which the integrals converge. These contact terms complicate the computation of 
amplitudes. The same was also shown in [28], where it was necessary to introduce these contact terms in order to have finite amplitudes at all momenta and for canceling certain boundary terms coming from divergences of unphysical states, which without the contact terms would render the theory non-unitary. Furthermore, it was argued that for some loop amplitudes the analytic continuation of the momenta was not possible, hence forcing the introduction of the contact terms.

The possible infinities in the amplitudes of the closed Green-Schwarz superstring have been analyzed in [29-32]. Furthermore, a prescription for the multi-loop amplitudes in Type II and heterotic superstrings has been constructed [33].

Because of the complications mentioned above, only several tree-level and one-loop amplitudes have been explicitly computed so far.

\section{The minimal pure spinor superstring}

\subsection{A review of the minimal pure spinor superstring}

The original version of the minimal pure spinor superstring was put forward by $\mathrm{N}$. Berkovits [34] (see [35] for a review) as a covariant manifestly target-space supersymmetric formulation of the superstring, which was more amenable to quantization than the Green-Schwarz superstring.

Concentrating on the holomorphic side the matter sigma-model is the one suggested by Siegel [36]

$$
S_{m}=\int d^{2} z\left(\frac{1}{2} \partial x^{m} \bar{\partial} x_{m}+p_{\alpha} \bar{\partial} \theta^{\alpha}\right),
$$

where $x^{m}(m=0, \ldots, 9)$ are the bosonic target-space coordinates, $\theta^{\alpha}$ are anti-commutative world-sheet scalars, which are target-space Weyl spinors $(\alpha=1, \ldots, 16)$ and the $p_{\alpha}$ are world-sheet 1 -forms which are the momenta conjugate to $\theta^{\alpha}$. To get the complete string one adds either a similar anti-holomorphic sector for Type II superstrings or the anti-holomorphic sector of the heterotic superstring. Originally this was to be supplanted by the Green-Schwarz constraint

$$
d_{\alpha}=p_{\alpha}+\gamma_{\alpha \beta}^{m} \partial x^{m} \theta^{\beta}+\frac{1}{2} \gamma_{\alpha \beta}^{m} \gamma_{m \gamma \delta} \theta^{\beta} \theta^{\gamma} \partial \theta^{\delta}=0
$$

However, it was suggested to relax this condition and have $p_{\alpha}$ as independent worldsheet field by adding a suitable set of first-class constraints.

The corresponding matter energy-momentum tensor is

$$
T_{m}=\frac{1}{2} \partial x^{m} \partial x_{m}+p_{\alpha} \partial \theta^{\alpha}
$$

from whose OPE one discovers that the central charge of the matter sector is -22 so it cannot form a consistent superstring alone. In order to remedy this, Berkovits 
added the fields $\lambda^{\alpha}$, which are world-sheet bosonic scalars and target-space spinors satisfying the pure spinor condition

$$
\lambda^{\alpha} \gamma_{\alpha \beta}^{m} \lambda^{\beta}=0
$$

and their conjugate momenta $w_{\alpha}$, which together form a constrained $\beta \gamma$-system. By solving the constraints one finds that only 11 of the $\lambda^{\alpha}$ are independent and 5 of the 16 components of $w_{\alpha}$ can be gauged away, leaving 11 components. Together they form a system of $11 \beta \gamma$-systems of weight $(1,0)$ having a central charge of 22 , which exactly cancels the central charge of the matter sector.

The Green-Schwarz constraints are replaced by the BRST operator

$$
Q_{B}=\oint \frac{d z}{2 \pi i} \lambda^{\alpha} d_{\alpha} .
$$

Although, this BRST symmetry does not seem to be the result of a straightforward gauge fixing it was shown that it is related to gauge fixing the Green-Schwarz superstring by a somewhat more involved process [37]. The nilpotence of the BRST operator is assured by the OPE

$$
d_{\alpha}(z) d_{\beta}(0) \sim \frac{2}{z} \gamma_{\alpha \beta}^{m} \Pi^{m}(0),
$$

because of which and the pure spinor constraint (4)

$$
Q_{B}^{2}=\oint \frac{d z}{2 \pi i} \lambda^{\alpha} \gamma_{\alpha \beta}^{m} \lambda^{\beta} \Pi_{m}(z)=0 .
$$

As usual, the physical spectrum of unintegrated vertex operators is given as the ghostnumber one (where $\lambda^{\alpha}$ and $w_{\alpha}$ carry ghost numbers 1 and -1 , respectively) cohomology of $Q_{B}$ and for the closed superstring the full vertex operator is the product of the left- and right-moving vertex operators.

The unintegrated massless vertex operators are thus of the form [34]

$$
U=\lambda^{\alpha} A_{\alpha}(x, \theta)
$$

The requirement that it be BRST-closed implies it must satisfy the equation

$$
\gamma_{m n p q r}^{\alpha \beta} D_{\alpha} A_{\beta}=0
$$

where $D_{\alpha}$ is the covariant supersymmetric derivative. The gauge transformation is $\delta U=Q_{B} \Omega(x, \theta)$, where $\Omega(x, \theta)$ is a ghost number zero superfield, so $\delta A_{\alpha}=D_{\alpha} \Omega$. The above equation and the gauge invariance can be showed to be the same as the field equations and gauge transformations of the $\mathscr{N}=1$ ten-dimensional Abelian pure gauge theory. Taking the product of two such vertex operators for the left- and right-movers one obtains the supergravity multiplet. The cohomology of the pure 
spinor superstring was shown to match that of the Green-Schwarz superstring in the light-cone gauge [38].

\subsection{The tree-level amplitude}

The prescription for the computation of tree-level amplitudes was originally deduced [34] and not derived from the path integral. Concentrating again on the left-moving sector, the scattering amplitude should include three unintegrated vertex operators because of the three holomorphic conformal Killing vectors on the sphere and the rest of the vertex operators should be in the integrated form. This suggests that the saturation rule for the tree-level scattering amplitude should have ghost number three. Hence, the scattering amplitude for $N$ particles should be of the form

$$
\mathscr{A}=\left\langle\prod_{i=1}^{3} U_{i}\left(z_{i}\right) \prod_{j=4}^{N} \int d w_{j} V_{j}\left(w_{j}\right)\right\rangle,
$$

where $U_{i}$ are unintegrated ghost number one vertex operators and $V_{j}$ are integrated ghost number zero vertex operators.

The non-zero modes of the world-sheet fields are then integrated out by contracting them using their OPEs leaving an expression just with the zero-modes. The saturation rule for the zero modes must have several properties: it must have ghost number three, it should be BRST invariant so that the amplitudes are BRST invariant and it should be $S O(9,1)$ invariant in order to preserve the Lorentz invariance of the amplitudes and finally it must be supersymmetric. It turned out that the unique expression for the zero-mode saturation rule satisfying the above conditions [39] is

$$
\left\langle\left(\lambda \gamma^{m} \theta\right)\left(\lambda \gamma^{n} \theta\right)\left(\lambda \gamma^{p} \theta\right)\left(\theta \gamma_{m n p} \theta\right)\right\rangle=1
$$

Indeed it has been verified that the above prescription is independent of the choice of the three unintegrated vertex operators and that it reproduces the RNS tree-level amplitudes for the scattering of any number of bosonic massless vertex operators and up to four massless fermionic ones [40].

\subsection{Multi-loop amplitudes}

The computation of higher genus amplitudes required knowledge of the path integral measure. The required measure was found in [41]. The integration of the non-zero modes is straight forward and can be done either explicitly, or more easily by using their OPEs. One then has to perform the integration over the zero-modes.

Being a world-sheet scalar, $\lambda^{\alpha}$ has 11 zero-modes on a genus $g$ Riemann surfacethe number of its independent components. For the sphere the saturation rule can be written in the form

$$
\left\langle\mathscr{T}_{\left(\alpha_{1} \alpha_{2} \alpha_{3}\right)\left[\beta_{1} \ldots \beta_{5}\right]} \lambda^{\alpha_{1}} \lambda^{\alpha_{2}} \lambda^{\alpha_{3}} \theta^{\beta_{1}} \ldots \theta^{\beta_{5}}\right\rangle=1
$$


where $\mathscr{T}_{\left(\alpha_{1} \alpha_{2} \alpha_{3}\right)\left[\beta_{1} \ldots \beta_{5}\right]}$ is the unique Lorentz invariant tensor symmetric in its first three indices and antisymmetric in the last five which vanishes when two of its first three indices are contracted with a $\gamma$ matrix. The path integral measure [ $\mathscr{D} \lambda]$ for the zero-modes on a Riemann surface of arbitrary genus is defined by

$$
\left(d^{11} \lambda\right)^{\left[\alpha_{1} \ldots \alpha_{11}\right]}=[\mathscr{D} \lambda](\varepsilon \mathscr{T})_{\left(\beta_{1} \beta_{2} \beta_{3}\right)}^{\left[\alpha_{1} \ldots \alpha_{11}\right]} \lambda^{\beta_{1}} \lambda^{\beta_{2}} \lambda^{\beta_{3}}
$$

and $(\varepsilon \mathscr{T})_{\left(\beta_{1} \beta_{2} \beta_{3}\right)}^{\left[\alpha_{1} \ldots \alpha_{11}\right]}=\varepsilon^{\alpha_{1} \ldots \alpha_{16}} \mathscr{T}_{\left(\alpha_{1} \alpha_{2} \alpha_{3}\right)\left[\alpha_{12} \ldots \alpha_{16}\right]}$.

The pure spinor constraints generate a gauge symmetry $\delta w_{\alpha}=\lambda^{\beta} \gamma_{\alpha \beta}^{m} \Lambda^{m}$ so they appear in the vertex operators only through the gauge invariant Lorentz currents $N^{m n}=$ $\frac{1}{2} w_{\alpha} \gamma^{m n \alpha}{ }_{\beta} \lambda^{\beta}$ and the ghost current $J=w \lambda$. Hence, it is easier to integrate over them instead of over $w_{\alpha}$. It turns out that the Lorentz ghost currents are related by an identity [42] leaving just 11 independent currents - as one would expect from having just 11 independent $w_{\alpha}$. The path integral measure $[\mathscr{D} N]$ is defined by

$$
\left(d^{10} N\right)^{\left[\left[m_{1} n_{1}\right] \ldots\left[m_{10} n_{10}\right]\right]} \wedge d J=[\mathscr{D} N](\text { ghost number } 8 \text { term })
$$

(for the form of the ghost number 8 term consult [41]).

Thus, the naive genus $g$ amplitude computation prescription is

$$
\mathscr{A}=\int[\mathscr{D} \lambda] \prod_{i=1}^{g}[\mathscr{D} N] f\left(\lambda, N_{1}, J_{1}, \ldots, N_{g}, J_{g}\right),
$$

where $f\left(\lambda, N_{1}, J_{1}, \ldots, N_{g}, J_{g}\right)$ is the function of the zero-modes obtained by using the OPEs in order to integrate over the non-zero modes. However, this simplistic definition of the amplitudes diverges because of the integration over the unbounded zero-modes. In order to define the amplitude properly, picture changing operators must be introduced.

The picture changing operator for absorbing the zero-modes of the pure spinor is

$$
Y_{C}=C_{\alpha} \theta^{\alpha} \delta\left(C_{\beta} \lambda^{\beta}\right)
$$

where $C_{\alpha}$ is a constant Lorentz spinor, which is required since $\lambda$ and $\theta$ are not invariant under Lorentz transformations. Even though $Y_{C}$ is not manifestly Lorentz invariant since the constant spinor $C_{\alpha}$ seems to break this symmetry, it has been shown [41] that the Lorentz transformation of $Y_{C}$ is BRST-exact so it decouples from the amplitude, which remains Lorentz invariant. Additionally, the world-sheet derivative of $Y_{C}$ has been shown to be BRST-exact so the amplitude does not depend on the positions of the $Y_{C}$ insertions. The picture changing operators for the zero-modes of the Lorentz and ghost currents are similarly defined by

$$
Z_{B}=\frac{1}{2} B_{m n}\left(\lambda \gamma^{m n} d\right) \delta\left(B^{p q} N_{p q}\right), \quad Z_{J}=\left(\lambda^{\alpha} d_{\alpha}\right) \delta(J),
$$

where $B_{m n}$ is a constant antisymmetric tensor (which can be chosen such that the definition of $Z_{B}$ requires no regularization and it was also argued that $Z_{J}$ requires 
no regularization either). Once again the Lorentz transformations and the world-sheet derivatives of these picture changing operators are BRST-exact-assuring the amplitudes Lorentz invariance and independence of the position of the picture changing operators on the Riemann surface. $Z_{B}$ and $Z_{J}$ are also BRST-closed so the amplitude remains BRST invariant when they are inserted.

In order to have a finite genus $g$ amplitude it is required to have 11 insertions of $Y_{C}, 10 g$ insertions of $Z_{B}$ and $g$ insertions of $Z_{J}$ to absorb the bosonic zero-modes. It was verified that for the sphere this prescription indeed reproduces the saturation rule of tree-level amplitudes.

Since a genus $g$ Riemann surface has $3 g-3$ moduli which have to be integrated over, insertions of the $b$-ghost, i.e., a ghost satisfying $\{Q, b\}=T$, are also required. Unfortunately, no such ghost exists because it would have to be of ghost number -1 and $w_{\alpha}$, which is the only world-sheet field with such a ghost number, can only appear in the combinations $N^{m n}$ and $J$, which have ghost number zero. This obstacle can be circumvented by defining a non-local operator $\tilde{b}_{B}(u, z)$ satisfying

$$
\left\{Q, \tilde{b}_{B}(u, z)\right\}=T(u) Z_{B}(z) .
$$

(The form of $\tilde{b}_{B}$ is rather complicated, see [41] for details.). This substitute for the $b$-ghost can provide both the $3 g-3$ insertion of the $b$-ghost for the moduli and $3 g-3$ out of the $10 \mathrm{~g}$ insertions of $Z_{B}$.

At this stage all the building blocks for the computation of multi-loop amplitudes are available. The genus $g>1 \mathrm{~N}$-point amplitude for the closed string is given by the expression

$$
\begin{aligned}
\mathscr{A}= & \int \prod_{i=1}^{3 g-3} d^{2} \tau_{i}\langle| \prod_{p=1}^{3 g-3} \int d^{2} u_{p} \mu_{p}\left(u_{p}\right) \tilde{b}_{B_{p}}\left(u_{p}, z_{p}\right) \prod_{m=3 g-2}^{10 g} Z_{B_{m}}\left(z_{m}\right) \prod_{n=1}^{g} Z_{J}\left(v_{n}\right) \\
& \left.\times\left.\prod_{l=1}^{11} Y_{C_{l}}\left(y_{l}\right)\right|^{2} \prod_{k=1}^{N} \int d^{2} t_{k} U_{k}\left(t_{k}\right)\right\rangle
\end{aligned}
$$

where $\tau_{i}$ are the metric moduli, $\mu_{p}$ are the Beltrami differentials and $U_{k}$ are the integrated vertex operators.

The expressions for the amplitudes on the sphere and the torus are somewhat different because they also have some residual conformal symmetry that needs to be fixed by having some fixed unintegrated vertex operators. The amplitude computation prescription for sphere $(g=0)$ has already been given and the one for the torus $(g=1)$ has one metric modulus and one unintegrated vertex operator:

$$
\begin{aligned}
\mathscr{A}= & \int d^{2} \tau\left\langle\left|\int d^{2} u \mu(u) \tilde{b}_{B_{1}}\left(u, z_{1}\right) \prod_{p=2}^{10} Z_{B_{p}}\left(z_{p}\right) Z_{J}(v) \prod_{l=1}^{11} Y_{C_{l}}\right|^{2} V_{1}\left(t_{1}\right)\right. \\
& \left.\times \prod_{k=2}^{N} \int d^{2} t_{k} U_{k}\left(t_{k}\right)\right\rangle
\end{aligned}
$$

where $V_{1}$ is an unintegrated vertex operator. 


\subsection{Results and open questions}

Using the above prescription it was proven that genus $g$ massless $N$-point amplitudes vanish for $N<4$ and $g>0$ [41]. This proof was based on knowledge of the massless closed string vertex operators and the counting of zero-modes. With several additional assumptions this fact implies the finiteness of the amplitudes in the superstring perturbation theory through the vanishing of possibly diverging contributions to the amplitudes from the boundary of the moduli space.

The four-point massless amplitude was computed [41] and it was shown that the $R^{4}$ and $\partial^{2} R^{4}$ terms in the effective action are one-loop exact, i.e., they do not receive corrections higher than the one-loop one. This amplitude was claimed to match the Ramond-Neveu-Schwarz one for a constant field-strength in [43] and subtleties in the argument for this matching were addressed in [44].

The four-point amplitude for massless particles was computed to two loops using the minimal pure spinor multi-loop prescription [45]. Similarly to the vanishing proofs mentioned above, zero-mode counting arguments simplified the computation.

The two-loop amplitude of four Neveu-Schwarz states was shown to match the Ramond-Neveu-Schwarz one [46].

The pure spinor formalism (or more accurately, its superspace) was used to find some identities relating the kinematic factors of the tree-level, one-loop and two-loop massless four-point amplitudes [47].

The derivation of the multi-loop amplitude [41] was based on requiring the non-vanishing and finiteness of the path integration over the fermionic and bosonic zero-modes, from which the need for the picture changing operators was deduced. One would also like to be able to derive it from first principles (e.g., by gauge fixing as can be done in the bosonic string).

Finally, even though the pure spinor formulation has been related to the Green-Schwarz and RNS ones $[48,37]$ it seems there is not yet a consensus about its equivalence to the other formalisms.

\section{The non-minimal pure spinor superstring}

\subsection{A review of the non-minimal pure spinor superstring}

The non-minimal pure spinor superstring was proposed in 2005 [49] in order to address some of the inconveniences of the minimal pure spinor superstring such as the lack of manifest Lorentz invariance because of the need to pick a spinor and a tensor for the picture-changing operators as well as to yield a more conventional prescription for the computation of amplitudes.

In the non-minimal superstring one adds to the left-moving pure spinor $\lambda^{\alpha}$ and its conjugate momentum $w_{\alpha}$ the additional left-moving bosonic pure spinor $\bar{\lambda}_{\alpha}$ and the fermionic target-space spinor $r_{\alpha}$ (both are world-sheet scalars) subject to the constraints

$$
\bar{\lambda}_{\alpha} \gamma_{m}^{\alpha \beta} \bar{\lambda}_{\beta}=0, \quad \bar{\lambda}_{\alpha} \gamma_{m}^{\alpha \beta} r_{\beta}=0
$$


The non-minimal superstring action for the left-movers (this has to be supplemented by a suitable action for the right-movers) is now

$$
S=\int d^{2} z\left(\frac{1}{2} \partial x^{m} \bar{\partial} x_{m}+p_{\alpha} \bar{\partial} \theta^{\alpha}-w_{\alpha} \bar{\partial} \lambda^{\alpha}-\bar{w}^{\alpha} \bar{\partial} \bar{\lambda}_{\alpha}+s^{\alpha} \bar{\partial} r_{\alpha}\right),
$$

where the conjugate momenta $\bar{w}^{\alpha}$ and $s^{\alpha}$ have been introduced. It turns out that these additional fields do not modify the central charge of the theory so that it remains critical but they do change the ghost-number anomaly from -8 to 3 once the ghost current is redefined as $w_{\alpha} \lambda^{\alpha}-\bar{w}^{\alpha} \bar{\lambda}_{\alpha}$. This value of the ghost number anomaly allows the definition of the scattering amplitudes using the usual topological string methods [49].

In addition, the BRST operator is modified to

$$
Q=\int d z\left(\lambda^{\alpha} d_{\alpha}+\bar{w}^{\alpha} r_{\alpha}\right)
$$

The cohomology remains unaffected due to the quartet mechanism.

Although the minimal pure spinor superstring has no globally defined $b$-ghost satisfying $\{Q, \tilde{b}\}=T_{\min }$ with $T_{\min }$ being the energy-momentum tensor of the minimal pure spinor, such a ghost can be defined in the non-minimal pure spinor superstring. Using Čech cohomology language, it can be written as [50]

$$
\tilde{b}=\left(b_{\alpha}\right)+\left(b_{\alpha \beta}\right)+\left(b_{\alpha \beta \gamma}\right)+\left(b_{\alpha \beta \gamma \delta}\right),
$$

where if the pure spinor target-space is divided into patches $U_{\alpha}=\left\{\lambda \mid \lambda^{\alpha} \neq 0\right\}$ then $\left(b_{\alpha}\right)$ is defined on $U_{\alpha},\left(b_{\alpha \beta}\right)$ is defined on the intersection $U_{\alpha} \cap U_{\beta}$ and so on. They are found to be

$$
\begin{aligned}
\left(b_{\alpha}\right) & =\frac{G^{\alpha}}{\lambda^{\alpha}}, \\
\left(b_{\alpha \beta}\right) & =\frac{H^{[\alpha \beta]}}{\lambda^{\alpha} \lambda^{\beta}}, \\
\left(b_{\alpha \beta \gamma}\right) & =\frac{K^{[\alpha \beta \gamma]}}{\lambda^{\alpha} \lambda^{\beta} \lambda \gamma}, \\
\left(b_{\alpha \beta \gamma \delta}\right) & =\frac{L^{[\alpha \beta \gamma \delta]}}{\lambda^{\alpha} \lambda^{\beta} \lambda \gamma \lambda^{\delta}},
\end{aligned}
$$

where

$$
\begin{aligned}
\left\{Q, G^{\alpha}\right\} & =\lambda^{\alpha} T_{\min }, \quad\left[Q, H^{[\alpha \beta]}\right]=\lambda^{[\alpha} G^{\beta]}, \quad\left\{Q, K^{[\alpha \beta \gamma]}\right\}=\lambda^{[\alpha} H^{\beta \gamma]}, \\
{\left[Q, L^{[\alpha \beta \gamma \delta]}\right] } & =\lambda^{[\alpha} K^{\beta \gamma \delta]}, \quad \lambda^{[\alpha} L^{\beta \gamma \delta \sigma]}=0
\end{aligned}
$$

and the explicit expressions for $G^{\alpha}, H^{[\alpha \beta]}, K^{[\alpha \beta \gamma]}$ and $L^{[\alpha \beta \gamma \delta]}$ are given in [49]. 
In the Dolbeault picture this takes the form

$$
\tilde{b}=\frac{\bar{\lambda}_{\alpha} G^{\alpha}}{\bar{\lambda} \lambda}+\frac{\bar{\lambda}_{\alpha} r_{\beta} H^{[\alpha \beta]}}{(\bar{\lambda} \lambda)^{2}}-\frac{\bar{\lambda}_{\alpha} r_{\beta} r_{\gamma} K^{[\alpha \beta \gamma]}}{(\bar{\lambda} \lambda)^{3}}-\frac{\bar{\lambda}_{\alpha} r_{\beta} r_{\gamma} r_{\delta} L^{[\alpha \beta \gamma \delta]}}{(\bar{\lambda} \lambda)^{4}}
$$

This expression for $\tilde{b}$ satisfies $\{Q, \tilde{b}\}=T_{\min }$. Now, by defining the non-minimal $b$-ghost as

$$
b=\tilde{b}+s^{\alpha} \partial \bar{\lambda}_{\alpha}
$$

one indeed gets $\{Q, b\}=T$, where

$$
T=T_{\min }+\bar{w}^{\alpha} \partial \bar{\lambda}_{\alpha}-s^{\alpha} \partial r_{\alpha}
$$

is the energy-momentum tensor of the non-minimal pure spinor.

\subsection{Multi-loop amplitudes}

Since the ghost number anomaly 3 is that of the topological string, it is reasonable to use the usual prescription for the computation of scattering amplitudes $[49,50]$

$$
\mathscr{A}=\int_{\mathscr{M}_{g, N}} d^{3 g-3} \tau\left\langle\prod_{j=1}^{3 g-3}\left(\int d w_{j} \mu_{j}\left(w_{j}\right) b\left(w_{j}\right)\right) \prod_{r=1}^{N} \int d z_{r} U\left(z_{r}\right)\right\rangle
$$

where $\mathscr{M}_{g, N}$ is the moduli space of a genus $g$ Riemann surface with $N$ marked points. However, the above amplitude suffers from two problems. The first is that the integration over the 22 zero-modes of $\lambda$ and $\bar{\lambda}$ and the $22 \mathrm{~g}$ zero-modes of $w$ and $\bar{w}$ on a genus $g$ surface diverges. The second is that the $b$-ghost (28) contains inverse powers of $\bar{\lambda} \lambda$ so for a genus large enough divergences are expected from the $\bar{\lambda} \lambda \rightarrow 0$ region. This issue limited the original suggestion [49] to amplitudes with $g<3$.

The first problem is solved by the fact that after an appropriate regularization the zeros due to the integration over the fermionic zero-modes of $\theta^{\alpha}, r_{\alpha}, p_{\alpha}$ and $s^{\alpha}$ cancel the divergence generated by the integration over the bosonic zero-modes of $\lambda^{\alpha}, \bar{\lambda}_{\alpha}$, $w_{\alpha}$ and $\bar{w}^{\alpha}$. In order to see that a regularization factor $\mathscr{N}=e^{\{Q, \chi\}}[50]$ with

$$
\chi=-\bar{\lambda}_{\alpha} \theta^{\alpha}-\sum_{I=1}^{g}\left(\frac{1}{2} N_{m n}^{I} S^{m n I}+J^{I} S^{I}\right)
$$

where $N_{m n}^{I}, S^{m n I}, J^{I}$ and $S^{I}$ are the zero-modes of the pure spinor minimal and nonminimal Lorentz currents and the minimal and non-minimal ghost currents, respectively (see [50] for the exact definitions of these currents), is introduced. This regulator should not change the value of the amplitude as it is of the form $\mathscr{N}=1+\{Q, \Omega\}$ for 
some $\Omega$ and hence the deviation from the identity decouples for scattering of physical states. This regulator provides a cut-off of the bosonic zero-modes integration as well as the fermionic insertions required to saturate the integration over the fermionic zero-modes.

The second problem arises from the fact that if the integrand of the path integral diverges as $(\bar{\lambda} \lambda)^{-11}$ or faster as $\bar{\lambda} \lambda \rightarrow 0$, the integration over the zero-modes will diverge. Since the $b$-ghost diverges as $(\bar{\lambda})^{-3}$ as $\bar{\lambda} \lambda \rightarrow 0$ and for a genus $g$ surface $3 g-3$ insertions of the $b$-ghost are required, the integral is expected to diverge for $g>2$.

In order to define the amplitude prescription to any order in the string perturbation theory, [50] suggested an appropriate BRST-invariant regularization for the $b$-ghost such that it no longer has a pole at $\bar{\lambda} \lambda \rightarrow 0$. This was done by defining the constant bosonic pure spinors $f^{\alpha}$ and $\bar{f}_{\alpha}$ and the constant fermions $g^{\alpha}, \bar{g}_{\alpha}$ subject to the constraints

$$
g^{\alpha} \gamma_{\alpha \beta}^{m} f^{\beta}=0, \quad \bar{g}_{\alpha} \gamma_{m}^{\alpha \beta} \bar{f}_{\beta}=0
$$

which transform under BRST transformations as

$$
\left[Q, f^{\alpha}\right]=0, \quad\left[Q, \bar{f}_{\alpha}\right]=\bar{g}_{\alpha}, \quad\left\{Q, g^{\alpha}\right\}=f^{\alpha}, \quad\left\{Q, \bar{g}_{\alpha}\right\}=0
$$

The regularized $b$-host is then defined as

$$
b_{\varepsilon}=\int d^{11} f d^{11} \bar{f} d^{11} g d^{11} \bar{g} e^{-\left(\bar{f}_{\alpha} f^{\alpha}+\bar{g}_{\alpha} g^{\alpha}\right)} b^{\prime},
$$

where $b^{\prime}$ is related to the $b$-ghost defined above by a similarity transformation of the form $b^{\prime}=e^{i \varepsilon S} b e^{-i \varepsilon S}$ with $S$ being BRST-exact (an interested reader should consult [50] for the details). Because of the form of the regularized $b$-ghost $b_{\varepsilon}=b+\left[Q, \chi_{\varepsilon}\right]$ the dependence on $\varepsilon$ is BRST-exact and thus decouples from amplitudes involving the scattering of physical states.

Therefore, the final form of the amplitude as suggested by [50] for $g \geq 2$ is

$$
\mathscr{A}=\lim _{\varepsilon \rightarrow 0} \int_{\mathscr{M}_{g, N}} d^{3 g-3} \tau\left\langle\mathscr{N} \prod_{i=1}^{3 g-3}\left(\int d w_{i} \mu_{i}\left(w_{i}\right) b_{\varepsilon}\left(w_{i}\right)\right) \prod_{j=1}^{N} \int d z_{j} U_{j}\left(z_{j}\right)\right\rangle
$$

It was further shown in [50] that F-terms, i.e., terms in the effective action which cannot be written as an integral over all the superspace fermionic coordinates, do not diverge even without the need to resort to regularization of the $b$-ghost. Other terms may require such regularization though. As a result, the computation of such F-terms is much simplified. 


\subsection{Results and open questions}

The non-minimal pure spinor formulation of the superstring facilitates the computation of multi-loop amplitudes compared with the minimal formulation because of the existence of a real (composite) $b$-ghost and the lack of need for picture changing operators, which makes the Lorentz invariance of the amplitudes manifest throughout the computation whereas in the minimal version Lorentz invariance is only up to BRST-exact terms.

The prescription given in [50] appears to be finite. However, its consistency requires that no operators with poles higher than tenth order in $\bar{\lambda} \lambda$ are allowed in the cohomology. This requirement is essential both for the finiteness of the amplitudes as well as for assuring the non-triviality of the cohomology since if operators with higher poles are permitted, there exists a homotopy operator, which renders the cohomology trivial [49]. This is an ad hoc requirement, which does not arise naturally in the theory.

Using the non-minimal pure spinor formalism it was proven that terms of the form $\partial^{n} R^{4}$ in the effective actions of the Type II superstrings do not receive perturbative corrections higher than $n / 2$ loops for $0<n<12$ [51]. It was further shown there that such terms are identical in the Type IIA and Type IIB effective actions for $n<9$.

The open string massless four-point amplitudes were computed up to two loops using the non-minimal pure spinor formulation and were shown to be the same as the corresponding results obtained using the minimal version of the pure spinor superstring [52], lending credibility to the belief that the two versions of the pure spinor superstring are equivalent. The anomaly in the six-point amplitude in the Type I superstring was also computed using the non-minimal formalism [52].

There is yet no proof of the equivalence of the minimal and non-minimal pure spinor formulations. This issue might be difficult to tackle because of the large differences in the amplitude computation prescriptions of the two.

\section{Summary}

We briefly reviewed the status of superstring perturbation theory in the various superstring formulations and described in more detail perturbation theory for the pure spinor formulation. It seems that the latter is more developed then the former ones and is much more tractable. It has also been put to use for proving various vanishing and non-renormalization theorems in a relatively simple manner. However, there are still open questions regarding the pure spinor superstring and the rigorous derivation of the amplitude prescription from first principles. Meanwhile, perturbation theory in RNS is still an active area of research and one may expect more progress in the future.

Open Access This article is distributed under the terms of the Creative Commons Attribution Noncommercial License which permits any noncommercial use, distribution, and reproduction in any medium, provided the original author(s) and source are credited.

\section{References}

1. Polchinski, J.: String theory. In: Superstring Theory and Beyond, p. 531 Cambridge University Press, Cambridge (1998) 
2. D’Hoker, E., Phong, D.H.: Loop Amplitudes for the Fermionic String. Nucl. Phys. B 278, 225 (1986)

3. Moore, G.W., Nelson, P.C., Polchinski, J.: Strings and Supermoduli. Phys. Lett. B 169, 47 (1986)

4. Aoki, K., D'Hoker, E., Phong, D.H.: Unitarity of closed superstring perturbation theory. Nucl. Phys. B 342, 149-230 (1990)

5. D'Hoker, E., Phong, D.H.: Momentum analyticity and finiteness of the one loop superstring amplitude. Phys. Rev. Lett. 70, 3692-3695 (1993)

6. D'Hoker, E., Phong, D.H.: The geometry of string perturbation theory. Rev. Mod. Phys. 60, 917 (1998)

7. D'Hoker, E., Phong, D.H.: Conformal scalar fields and chiral splitting on superriemann surfaces. Commun. Math. Phys. 125, 469 (1989)

8. D’Hoker, E., Phong, D.H.: Two-loop superstrings I, main formulas. Phys. Lett. B 529, 241-255 (2002)

9. D’Hoker, E., Phong, D.H.: Two-loop superstrings II, the chiral measure on moduli space. Nucl. Phys. B 636, 3-60 (2002)

10. D'Hoker, E., Phong, D.H.: Two-loop superstrings III, slice independence and absence of ambiguities. Nucl. Phys. B 636, 61-79 (2002)

11. D'Hoker, E., Phong, D.H.: Two-loop superstrings IV, the cosmological constant and modular forms. Nucl. Phys. B 639, 129-181 (2002)

12. D'Hoker, E., Phong, D.H.: Two-loop superstrings V: gauge slice independence of the N-point function. Nucl. Phys. B 715, 91-119 (2005)

13. D'Hoker, E., Phong, D.H.: Two-loop superstrings VI: non-renormalization theorems and the 4-point function. Nucl. Phys. B 715, 3-90 (2005)

14. D'Hoker, E., Phong, D.H.: Asyzygies, modular forms, and the superstring measure, I. Nucl. Phys. B 710, 58-82 (2005)

15. D'Hoker, E., Phong, D.H.: Asyzygies, modular forms, and the superstring measure, II. Nucl. Phys. B 710, 83-116 (2005)

16. Cacciatori, S.L., Piazza, F.D., van Geemen, B.: Modular forms and three loop superstring amplitudes. Nucl. Phys. B 800, 565-590 (2008)

17. Matone, M., Volpato, R.: Higher genus superstring amplitudes from the geometry of moduli spaces. Nucl. Phys. B 732, 321-340 (2006)

18. Grushevsky S.: Superstring scattering amplitudes in higher genus. (2008)

19. Morozov, A.: NSR measures on hyperelliptic locus and non-renormalization of 1, 2, 3-point functions. Phys. Lett. B 664, 116-122 (2008)

20. Matone M., Volpato R.: Superstring measure and non-renormalization of the three-point amplitude. (2008)

21. Grushevsky S., Manni R.S.: On the cosmological constant for the chiral superstring measure (2008)

22. Green, M.B., Schwarz, J.H.: Superstring interactions. Nucl. Phys. B 218, 43-88 (1983)

23. Green, M.B., Schwarz, J.H., Brink, L.: Superfield theory of type II superstrings. Nucl. Phys. B 219, 437478 (1983)

24. Green, M.B., Schwarz, J.H.: Superstring field theory. Nucl. Phys. B 243, 475-536 (1984)

25. Greensite, J., Klinkhamer, F.R.: New interactions for superstrings. Nucl. Phys. B 281, 269 (1987)

26. Greensite, J., Klinkhamer, F.R.: Contact interactions in closed superstring field theory. Nucl. Phys. B 291, 557 (1987)

27. Greensite, J., Klinkhamer, F.R.: Superstring amplitudes and contact interactions. Nucl. Phys. B 304, 108 (1988)

28. Green, M.B., Seiberg, N.: Contact interactions in superstring thoery. Nucl. Phys. B 299, 559 (1988)

29. Restuccia, A., Taylor, J.G.: Finiteness of type II superstring amplitudes. Phys. Lett. B 187, 267 (1987)

30. Taylor, J.G., Restuccia, A: Finiteness of heterotic superstring theories. Phys. Lett. B 187, 273 (1987)

31. Restuccia, A., Taylor, J.G.: On the infinites of closed superstring amplitude. Mod. Phys. Lett. A 3, 883 (1988)

32. Restuccia, A., Taylor, J.G.: Absence of divergences in type II and heterotic string multiloop amplitudes. Commun. Math. Phys. 112, 447 (1987)

33. Restuccia, A., Taylor, J.G.: The construction of multiloop superstring amplitudes in the light cone gauge. Phys. Rev. D36, 489 (1987)

34. Berkovits, N.: Super-poincare covariant quantization of the superstring. JHEP 04, 018 (2000)

35. Berkovits, N.: ICTP lectures on covariant quantization of the superstring (2002)

36. Siegel, W.: Classical superstring mechanics. Nucl. Phys. B 263, 93 (1986)

37. Berkovits, N.: Explaining the pure spinor formalism for the superstring. JHEP 01, 065 (2008)

38. Berkovits, N.: Cohomology in the pure spinor formalism for the superstring. JHEP 09, 046 (2000)

39. Berkovits, N.: Covariant quantization of the superstring. Nucl. Phys. Proc. Suppl. 127, 23-29 (2004) 
40. Berkovits, N., Vallilo, B.C.: Consistency of super-Poincare covariant superstring tree amplitudes. JHEP 07, 015 (2000)

41. Berkovits, N.: Multiloop amplitudes and vanishing theorems using the pure spinor formalism for the superstring. JHEP 09, 047 (2004)

42. Berkovits, N., Chandia, O.: Massive superstring vertex operator in $D=10$ superspace. JHEP 08, 040 (2002)

43. Anguelova, L., Grassi, P.A., Vanhove, P.: Covariant one-loop amplitudes in $D=11$. Nucl. Phys. B 702, 269-306 (2004)

44. Mafra, C.R.: Four-point one-loop amplitude computation in the pure spinor formalism. JHEP 01, 075 (2006)

45. Berkovits, N.: Super-poincare covariant two-loop superstring amplitudes. JHEP 01, 005 (2006)

46. Berkovits, N., Mafra, C.R.: Equivalence of two-loop superstring amplitudes in the pure spinor and RNS formalisms. . Phys. Rev. Lett. 96, 011602 (2006)

47. Mafra, C.R.: Pure spinor superspace identities for massless four-point kinematic factors. JHEP 04, 093 (2008)

48. Aisaka, Y., Kazama, Y.: Origin of pure spinor superstring. JHEP 05, 046 (2005)

49. Berkovits, N.: Pure spinor formalism as an $N=2$ topological string. JHEP 10, 089 (2005)

50. Berkovits, N., Nekrasov, N.: Multiloop superstring amplitudes from non-minimal pure spinor formalism. JHEP 12, 029 (2006)

51. Berkovits, N.: New higher-derivative R**4 theorems. Phys. Rev. Lett. 98, 211601 (2007)

52. Berkovits, N., Mafra, C.R.: Some superstring amplitude computations with the non-minimal pure spinor formalism. JHEP 11, 079 (2006) 